\title{
Influence of lead on growth and nutrient accumulation in Black gram (Vigna mungo. L)
}

\author{
S. Ravikumar*, P. Thamizhiniyzn \\ Department of Botany, Annamalai University, Annamalai Nagar - 609 002, India \\ *E-mail address: drsrk74@gmail.com
}

\begin{abstract}
This study was conducted to investigate toxic effects of $\mathrm{Pb}$ on growth and nutrient uptake in Black gram. Black gram was subjected to seven $\left(5,10,25,50,75,100 \& 200 \mathrm{~kg}^{-1}\right)$ levels of lead. Due to $\mathrm{Pb}$ toxicity, plant growth was adversely affected and relatively a severe reduction in root biomass was recorded. The $\mathrm{Pb}$ accumulation increased with the increase in lead concentrations. The uptake of different nutrients, i.e., $\mathrm{N}, \mathrm{P}, \mathrm{K}, \mathrm{Ca}$ and $\mathrm{Mg}$ was reduced in black gram due to the lead treatment.
\end{abstract}

Keyword: Lead; Black gram; Nutrients; Growth; Dry weight

\section{INTRODUCTION}

Although the major causes of accumulation of high levels of heavy metals in soils are a variety of man-made activities including manufacturing, agricultural, mining, and waste removal practices (Uwah et al., 2009) they are also brought due to the use of metal-enriched fertilizers and pesticides (Nouri et al., 2008). These metal ions dissolved in irrigation water contaminate the cultivated soils and can have toxic impact on living system, if present in excessive amounts (Nriago,1990). Lead $(\mathrm{Pb})$ is one of the most important heavy metals frequently available in the environment and its most common sources are vehicles and automobiles (Sezgin et al., 2003).In plants, its accumulation has been reported in stem, leaves, roots and seeds, which increases with increase in $\mathrm{Pb}$ levels in the growth medium (Yilmaz et al., 2009). It detrimentally influences plant growth (Wang et al., 2007) by hampering a variety of physiological processes including nutrient uptake (Sinha et al., 2006)

Plant injuries caused by $\mathrm{Pb}$ are very frequent and drastic, especially on the rooting system, which results into severe reduction in plant productivity (Uveges et al., 2002). Lead toxicity alters the normal metabolic pathways in plants including photosynthesis, respiration, and other such key metabolic processes by disrupting specific cellular enzymes (Ruley et al., 2004). Crop yield is also reduced due to $\mathrm{Pb}$-induced inhibition in metabolic processes in plants. Since most crops can frequently accumulate high levels of $\mathrm{Pb}$, they serve as a source of heavy metal supply in the food of humans and animals which ultimately cause health hazards in them. So, an attempt were made on the impact of Lead on Black gram growth and nutrient accumulation. 


\section{MATERIALS AND METHODS}

Black gram (Vigna mungo (L) Hepper) seeds were obtained from the Regional Rice Research station, Aaduthurai, Tamil Nadu, India. Uniform seeds were selected and surface sterilized with $0.01 \% \mathrm{HgCl}_{2}$ solution for 2 minutes. Uniform sized pots were filled with three kilograms soil and mixed with different concentrations (control, 5, 10, 25, 50, 75, 100 and $200 \mathrm{mg} \mathrm{kg}^{-1}$ soil) of Lead. Three replicates were maintained. Plant Growth were measured by using centimeter scale and recorded. The same plant samples were taken for morphological studies were also used for the determination of dry weight by using electrical single pan balance. Their dry weights were determined by keeping the plant materials in a hot air oven at $80{ }^{\circ} \mathrm{C}$ for $24 \mathrm{hrs}$ and recorded. Similarly the accumulation of Lead was measured followed by the method of Piper, 1966 and the mineral elements such as Total nitrogen (Jackson, 1958), Phosphorus (Black, 1965), Potassium (Williams and Twine, 1960), Calcium and magnesium (Yoshida et al., 1972)was also measured.

\section{RESULTS AND DISCUSSION}

Due to the application of $\mathrm{Pb}$, Plant growth and biomass was significantly reduced in Blackgram (Table 1). However, the most severe effect was noted on roots and shoots of plants growing under the highest level of $\mathrm{Pb}\left(200 \mathrm{mg} \mathrm{kg}^{-1}\right.$ soil). The effect of $\mathrm{Pb}$ toxicity was more severe on roots than on shoots in blackgram both in shoots and roots increased with increase in external $\mathrm{Pb}$ level. Lead accumulation in the roots was higher than that in the shoots.

In the present study, plant growth, root and shoot biomass was reduced due to $\mathrm{Pb}$ toxicity in Blackgram. However, the decrease in root biomass was more marked than that in shoot biomass. It is now widely known that heavy metals in soil or water can adversely affect the growth and ionic concentration in different tissues of plants (Sanchez et al., 1999; Ali et al., 2009). As the roots contained higher amount of $\mathrm{Pb}$ than that of shoots, which might have antagonistically disturbed the uptake of essential nutrients and water absorption resulting into reduced root growth, as observed in the present investigation.

The nutrient contents in the shoots and roots were imbalanced by the high quantity of $\mathrm{Pb}$ present in the soil. Essential macro- nutrients like $\mathrm{N}, \mathrm{P}$ and $\mathrm{K}$ in the shoots decreased with increased levels of $\mathrm{Pb}$ from 0 to $200 \mathrm{mg} \mathrm{Pb} \mathrm{kg}{ }^{-1}$ soil, but reduction in $\mathrm{K}$ and $\mathrm{N}$ was more marked in the roots of blackgram (Table 2). This clearly shows that $\mathrm{Pb}$ presence in the growth medium inhibited the absorption of $\mathrm{N}$ and $\mathrm{K}$ as already observed in sugar beet (Larbi et al., 2002). As these both elements are mobile so whatever quantity of $\mathrm{K}$ and $\mathrm{N}$ is absorbed by the roots, it is ultimately translocated to the shoot, because these nutrients are required in large amount to maintain the plant metabolic activities (Kaya et al., 2003). Although P content decreased significantly in both shoots and roots, the roots accumulated more amount of $P$ than that of shoots. It has been earlier reported that nutrient uptake by roots also depends on the plasma membrane selective properties. Thus $\mathrm{Pb}$ may perturb the normal uptake of nutrients by changing the permeability of the plasma membrane as well as by affecting all the processes involved in nutrient transport across the membrane (Gussarsson, 1994). Calcium and Magnesium concentrations in the roots were increased slightly due to increase in external $\mathrm{Pb}$ from 0 to $200 \mathrm{mg} \mathrm{kg}^{-1}$ of soil, while their amount was significantly decreased in the shoots of blackgram (Table 3). Since $\mathrm{Ca}$ is an integral component of cell wall as well as it is usually bound to the exterior surface of the plasma membrane, it plays a vital role in maintaining the integrity of both cell wall and membrane ( Wensheng et al., 1997). An increase in Ca 
concentration under $\mathrm{Pb}$ contamination in the roots could be a putatitve mechanism of minimizing the toxic effects of $\mathrm{Pb}$ and a decrease in $\mathrm{Ca}$ concentration in the shoots under $\mathrm{Pb}$ toxicity may have been an indication of a damaged intercellular defense system. Magnesium contents decreased in the shoots while they increased in the roots due to $\mathrm{Pb}$ application. This suggests that uptake of $\mathrm{Mg}$ was not affected due to $\mathrm{Pb}$ present in the growth medium; however, its translocation to the shoots was inhibited. The reduction in shoot $\mathrm{Mg}$ might have been one of the factors for causing reduction in chlorophyll content (Wensheng et al., 1997)..

Table 1. Effect of Lead on the Plant height $(\mathrm{cm} / \mathrm{p})$ and Dry weight $(\mathrm{g} / \mathrm{p})$ of root and shoot of Black gram (Vigna mungo (L) Merr).

\begin{tabular}{|c|c|c|c|c|c|c|c|c|c|}
\hline \multirow{2}{*}{$\begin{array}{l}\text { Lead } \\
\text { conc. } \\
(\mathrm{mg} / \mathrm{kg})\end{array}$} & \multicolumn{3}{|c|}{ Plant height } & \multicolumn{3}{|c|}{ Dry weight in Shoot } & \multicolumn{3}{|c|}{ Dry weight in Root } \\
\hline & 20 & 40 & 60 & 20 & 40 & 60 & 20 & 40 & 60 \\
\hline Control & 30.621 & 42.354 & 50.712 & 0.927 & 1.478 & 1.826 & 0.517 & 0.718 & 0.847 \\
\hline 10 & $\begin{array}{c}27.854 \\
(-9.036)\end{array}$ & $\begin{array}{l}39.218 \\
(-7.404)\end{array}$ & $\begin{array}{c}45.422 \\
(-10.431)\end{array}$ & $\begin{array}{c}0.812 \\
(-12.405)\end{array}$ & $\begin{array}{c}1.117 \\
(-24.424)\end{array}$ & $\begin{array}{c}1.427 \\
(-21.851)\end{array}$ & $\begin{array}{c}0.474 \\
(-8.317)\end{array}$ & $\begin{array}{c}0.624 \\
(-13.091)\end{array}$ & $\begin{array}{c}0.785 \\
(-7.319)\end{array}$ \\
\hline 25 & $\begin{array}{c}25.527 \\
(-16.635)\end{array}$ & $\begin{array}{c}34.422 \\
(-18.727)\end{array}$ & $\begin{array}{c}42.495 \\
(-16.203)\end{array}$ & $\begin{array}{c}0.754 \\
(-18.662)\end{array}$ & $\begin{array}{c}1.056 \\
(-28.552)\end{array}$ & $\begin{array}{c}1.218 \\
(-33.296)\end{array}$ & $\begin{array}{c}0.437 \\
(-15.473)\end{array}$ & $\begin{array}{c}0.611 \\
(-14.902)\end{array}$ & $\begin{array}{c}0.744 \\
(-12.160)\end{array}$ \\
\hline 50 & $\begin{array}{c}21.396 \\
(-30.128)\end{array}$ & $\begin{array}{c}30.139 \\
(-28.840)\end{array}$ & $\begin{array}{c}39.854 \\
(-21.411)\end{array}$ & $\begin{array}{c}0.622 \\
(-13.901)\end{array}$ & $\begin{array}{c}0.827 \\
(-44.046)\end{array}$ & $\begin{array}{c}1.147 \\
(-37.188)\end{array}$ & $\begin{array}{c}0.396 \\
(-23.404)\end{array}$ & $\begin{array}{c}0.575 \\
(-19.916)\end{array}$ & $\begin{array}{c}0.695 \\
(-17.945)\end{array}$ \\
\hline 75 & $\begin{array}{c}17.412 \\
(-43.137)\end{array}$ & $\begin{array}{c}26.622 \\
(-37.144)\end{array}$ & $\begin{array}{c}36.217 \\
(-28.582)\end{array}$ & $\begin{array}{c}0.506 \\
(-45.415)\end{array}$ & $\begin{array}{c}0.796 \\
(-46.143)\end{array}$ & $\begin{array}{c}0.932 \\
(-48.959)\end{array}$ & $\begin{array}{c}0.352 \\
(-31.914)\end{array}$ & $\begin{array}{c}0.527 \\
(-26.601)\end{array}$ & $\begin{array}{c}0.627 \\
(-25.974)\end{array}$ \\
\hline 100 & $\begin{array}{c}14.396 \\
(-52.986)\end{array}$ & $\begin{array}{c}25.363 \\
(-40.116)\end{array}$ & $\begin{array}{c}33.422 \\
(-34.094)\end{array}$ & $\begin{array}{c}0.417 \\
(-55.016)\end{array}$ & $\begin{array}{c}0.725 \\
(-50.947)\end{array}$ & $\begin{array}{c}0.754 \\
(-58.707)\end{array}$ & $\begin{array}{c}0.317 \\
(-38.684)\end{array}$ & $\begin{array}{c}0.491 \\
(-31.615)\end{array}$ & $\begin{array}{c}0.594 \\
(-29.870)\end{array}$ \\
\hline 200 & $\begin{array}{c}10.448 \\
(-65.879)\end{array}$ & $\begin{array}{c}21.125 \\
(-50.134)\end{array}$ & $\begin{array}{c}31.398 \\
(-38.085)\end{array}$ & $\begin{array}{c}0.325 \\
(-64.940)\end{array}$ & $\begin{array}{c}0.628 \\
(-57.510)\end{array}$ & $\begin{array}{c}0.628 \\
(-65.607)\end{array}$ & $\begin{array}{c}0.298 \\
(-42.359)\end{array}$ & $\begin{array}{c}0.452 \\
(-37.047)\end{array}$ & $\begin{array}{c}0.544 \\
(-35.773)\end{array}$ \\
\hline
\end{tabular}

Percentage over control is expressed in parentheses

F values are significant at $1 \%$ level.

Table 2. Effect of Lead on accumulation of Nitrogen (ppm), Phosphorus (ppm) and Potassium (ppm) content of Black gram (Vigna mungo (L) Merr).

\begin{tabular}{|c|c|c|c|c|c|c|c|c|c|}
\hline \multirow{2}{*}{$\begin{array}{l}\text { Lead } \\
\text { conc. } \\
(\mathrm{mg} / \mathrm{kg})\end{array}$} & \multicolumn{3}{|c|}{ Nitrogen } & \multicolumn{3}{|c|}{ Phosphorus } & \multicolumn{3}{|c|}{ Potassium } \\
\hline & 20 & 40 & 60 & 20 & 40 & 60 & 20 & 40 & 60 \\
\hline Control & 162.46 & 176.82 & 167.42 & 23.62 & 37.91 & 26.10 & 105.41 & 162.32 & 112.37 \\
\hline
\end{tabular}




\begin{tabular}{|c|c|c|c|c|c|c|c|c|c|}
\hline 10 & $\begin{array}{l}151.62 \\
(-6.67)\end{array}$ & $\begin{array}{l}161.59 \\
(-8.61)\end{array}$ & $\begin{array}{l}152.81 \\
(-8.60)\end{array}$ & $\begin{array}{c}20.74 \\
(-15.31)\end{array}$ & $\begin{array}{c}34.39 \\
(-9.28)\end{array}$ & $\begin{array}{c}22.63 \\
(-13.29)\end{array}$ & $\begin{array}{c}98.36 \\
(-6.68)\end{array}$ & $\begin{array}{c}137.32 \\
(-15.40)\end{array}$ & $\begin{array}{l}102.29 \\
(-8.97)\end{array}$ \\
\hline 25 & $\begin{array}{l}147.62 \\
(-9.13)\end{array}$ & $\begin{array}{c}158.76 \\
(-10.21)\end{array}$ & $\begin{array}{c}148.75 \\
(-11.15)\end{array}$ & $\begin{array}{c}18.37 \\
(-22.22)\end{array}$ & $\begin{array}{c}32.92 \\
(-13.16)\end{array}$ & $\begin{array}{c}20.55 \\
(-21.26)\end{array}$ & $\begin{array}{c}88.96 \\
(-16.80)\end{array}$ & $\begin{array}{c}126.51 \\
(-22.06)\end{array}$ & $\begin{array}{c}89.65 \\
(-20.21)\end{array}$ \\
\hline 50 & $\begin{array}{c}138.82 \\
(-14.55)\end{array}$ & $\begin{array}{c}152.39 \\
(-13.81)\end{array}$ & $\begin{array}{c}139.82 \\
(-16.48)\end{array}$ & $\begin{array}{c}15.92 \\
(-32.59)\end{array}$ & $\begin{array}{c}28.76 \\
(-24.31)\end{array}$ & $\begin{array}{c}17.92 \\
(-31.34)\end{array}$ & $\begin{array}{c}81.82 \\
(-22.37)\end{array}$ & $\begin{array}{c}110.52 \\
(-31.91)\end{array}$ & $\begin{array}{c}83.27 \\
(-25.89)\end{array}$ \\
\hline 75 & $\begin{array}{c}132.65 \\
(-18.34)\end{array}$ & $\begin{array}{c}144.28 \\
(-18.40)\end{array}$ & $\begin{array}{c}130.49 \\
(-22.05)\end{array}$ & $\begin{array}{c}14.43 \\
(-38.90)\end{array}$ & $\begin{array}{c}26.95 \\
(-28.91)\end{array}$ & $\begin{array}{c}15.56 \\
(-40.38)\end{array}$ & $\begin{array}{c}72.39 \\
(-31.32)\end{array}$ & $\begin{array}{c}104.37 \\
(-35.70)\end{array}$ & $\begin{array}{c}74.52 \\
(-33.68)\end{array}$ \\
\hline 100 & $\begin{array}{c}126.46 \\
(-22.15)\end{array}$ & $\begin{array}{c}141.38 \\
(-20.04)\end{array}$ & $\begin{array}{c}121.52 \\
(-27.41)\end{array}$ & $\begin{array}{c}12.87 \\
(-45.51)\end{array}$ & $\begin{array}{c}24.48 \\
(-35.42)\end{array}$ & $\begin{array}{c}12.39 \\
(-52.52)\end{array}$ & $\begin{array}{c}58.62 \\
(-44.38)\end{array}$ & $\begin{array}{c}96.82 \\
(-40.35)\end{array}$ & $\begin{array}{c}70.32 \\
(-37.42)\end{array}$ \\
\hline 200 & $\begin{array}{c}119.51 \\
(-26.43)\end{array}$ & $\begin{array}{c}135.46 \\
(-23.39)\end{array}$ & $\begin{array}{c}103.39 \\
(-38.24)\end{array}$ & $\begin{array}{c}10.42 \\
(-55.88)\end{array}$ & $\begin{array}{c}21.52 \\
(-42.23)\end{array}$ & $\begin{array}{c}10.79 \\
(-58.65)\end{array}$ & $\begin{array}{c}46.37 \\
(-56.09)\end{array}$ & $\begin{array}{c}87.65 \\
(-46.00)\end{array}$ & $\begin{array}{c}65.46 \\
(-41.74)\end{array}$ \\
\hline
\end{tabular}

Percentage over control is expressed in parentheses

$\mathrm{F}$ values are significant at $1 \%$ level.

Table 3. Effect of Lead on accumulation of Calcium (ppm), Magnesium (ppm) and Lead (ppm) content of Black gram (Vigna mungo (L) Merr).

\begin{tabular}{|c|c|c|c|c|c|c|c|c|c|}
\hline \multirow{2}{*}{$\begin{array}{c}\text { Lead } \\
\text { conc. } \\
(\mathrm{mg} / \mathrm{kg})\end{array}$} & \multicolumn{3}{|c|}{ Calcium } & \multicolumn{3}{|c|}{ Magnesium } & \multicolumn{3}{|c|}{ Lead } \\
\hline & 20 & 40 & 60 & 20 & 40 & 60 & 20 & 40 & 60 \\
\hline Control & 52.36 & 59.46 & 55.42 & 34.56 & 48.32 & 34.92 & 43.41 & 88.32 & 122.37 \\
\hline 10 & $\begin{array}{c}49.52 \\
(-5.233)\end{array}$ & $\begin{array}{c}54.82 \\
(-7.803)\end{array}$ & $\begin{array}{c}52.92 \\
(-4.511)\end{array}$ & $\begin{array}{c}31.41 \\
(-9.11)\end{array}$ & $\begin{array}{c}44.26 \\
(-8.40)\end{array}$ & $\begin{array}{c}31.16 \\
(-10.76)\end{array}$ & $\begin{array}{c}65.36 \\
(-6.68)\end{array}$ & $\begin{array}{c}97.32 \\
(-15.40)\end{array}$ & $\begin{array}{l}132.29 \\
(-8.97)\end{array}$ \\
\hline 25 & $\begin{array}{c}44.37 \\
(-15.259)\end{array}$ & $\begin{array}{c}49.37 \\
(-16.969)\end{array}$ & $\begin{array}{c}46.35 \\
(-16.365)\end{array}$ & $\begin{array}{c}28.39 \\
(-17.85)\end{array}$ & $\begin{array}{c}40.46 \\
(-16.26)\end{array}$ & $\begin{array}{c}28.34 \\
(-18.84)\end{array}$ & $\begin{array}{c}78.96 \\
(-16.80)\end{array}$ & $\begin{array}{c}111.51 \\
(-22.06)\end{array}$ & $\begin{array}{c}149.65 \\
(-20.21)\end{array}$ \\
\hline 50 & $\begin{array}{c}38.62 \\
(-26.241)\end{array}$ & $\begin{array}{c}42.27 \\
(-28.910)\end{array}$ & $\begin{array}{c}40.39 \\
(-27.120)\end{array}$ & $\begin{array}{c}25.52 \\
(-26.15)\end{array}$ & $\begin{array}{c}36.29 \\
(-24.89)\end{array}$ & $\begin{array}{c}27.56 \\
(-21.07)\end{array}$ & $\begin{array}{c}91.82 \\
(-22.37)\end{array}$ & $\begin{array}{c}121.52 \\
(-31.91)\end{array}$ & $\begin{array}{c}153.27 \\
(-25.89)\end{array}$ \\
\hline 75 & $\begin{array}{c}31.49 \\
(-39.858)\end{array}$ & $\begin{array}{c}35.62 \\
(-40.090)\end{array}$ & $\begin{array}{c}33.36 \\
(-39.805)\end{array}$ & $\begin{array}{c}22.96 \\
(-33.56)\end{array}$ & $\begin{array}{c}34.82 \\
(-27.93)\end{array}$ & $\begin{array}{c}24.83 \\
(-28.89)\end{array}$ & $\begin{array}{c}112.39 \\
(-31.32)\end{array}$ & $\begin{array}{c}132.37 \\
(-35.70)\end{array}$ & $\begin{array}{c}174.52 \\
(-33.68)\end{array}$ \\
\hline
\end{tabular}




\begin{tabular}{|c|c|c|c|c|c|c|c|c|c|}
\hline 100 & $\begin{array}{c}24.72 \\
(-52.788)\end{array}$ & $\begin{array}{c}29.82 \\
(-49.848)\end{array}$ & $\begin{array}{l}27.62 \\
(-50.162)\end{array}$ & $\begin{array}{c}20.84 \\
(-39.69)\end{array}$ & $\begin{array}{c}31.62 \\
(-34.56)\end{array}$ & $\begin{array}{c}21.49 \\
(-38.45)\end{array}$ & $\begin{array}{c}138.62 \\
(-44.38)\end{array}$ & $\begin{array}{c}146.82 \\
(-40.35)\end{array}$ & $\begin{array}{c}200.32 \\
(-37.42)\end{array}$ \\
\hline 200 & $\begin{array}{c}19.56 \\
(-62.643)\end{array}$ & $\begin{array}{c}26.31 \\
(-55.751)\end{array}$ & $\begin{array}{c}21.82 \\
(-60.627)\end{array}$ & $\begin{array}{c}16.39 \\
(-52.57)\end{array}$ & $\begin{array}{c}28.72 \\
(-40.56)\end{array}$ & $\begin{array}{c}18.24 \\
(-47.76)\end{array}$ & $\begin{array}{c}146.37 \\
(-56.09)\end{array}$ & $\begin{array}{c}187.65 \\
(-46.00)\end{array}$ & $\begin{array}{c}265.46 \\
(-41.74)\end{array}$ \\
\hline
\end{tabular}

Percentage over control is expressed in parentheses

$\mathrm{F}$ values are significant at $1 \%$ level.

\section{CONCLUSION}

From the present investigation, it can be concluded that the level of Lead above 200 $\mathrm{mg} / \mathrm{l}$ is proved to be lethal to Black gram. However, Lead contaminated water can be properly treated and then discharged into nearby water bodies in order to prevent water pollution. Both government and public sector should join hands in the creation of a clean and green environment.

\section{Acknowledgements}

The authors are thankful to the Professor and Head, Department of Botany, Annamalai University, Annamalai Nagar, Tamil Nadu, India.

\section{References}

[1] Ali M.A., M. Ashraf, H.R. Athar, J. Hazar. Mater 172 (2009) 964-969.

[2] Black C.A., 1965. Methods of soil analysis part 2, Inc., Madison, W. Scocini

[3] Gussarsson M., J. Plant Nut 17 (1994) 2151-2163.

[4] Jackson M.L. (1958). In: Soil chemical analysis. Prentice-Hall of India, Private Limited, New Delhi

[5] Kaya C., B.E. Ak, D. Higgs, J. Plant Nutr. 26 (2003) 543-560.

[6] Larbi A., F. Morals A. Abadia, Y. Geogrocena J. J. Llucena, J. Abadia, Funct Plant Biol. 29 (2002) 1453-1464.

[7] Nouri J., A.H. Mahvi, G.R. Jahed, A. Babaei, Environ. Geol. 55 (2008) 1337-1343.

[8] Nriago J. O., Environment 32 (1990) 7-33.

[9] Piper C., (1966). Soil and plant analysis. Asian Hans Publishers, Bombay, pp. 11-36.

[10] Ruley A.T., N.C. Sharma, S.V. Sahi, Plant Physiol Biochem. 42 (2004) 899-906.

[11] Sanchez P.G., L.P. Fernandez, L.T. Trejo, G.G. Aleantra, J.D. Cruz, Acta Hort 481 (1999) 617-623.

[12] Sezgin N., H.K. Ozcan, G. Demir, S. Nemlioglu, C. Bayat, Environ. Intl. 29 (2003) 979- 985. 
[13] Sinha P., B.K. Dube, P. Srivastava, C. Chatterjee, Chemosphere 65 (2006) 651-656.

[14] Uveges J.L., A.L. Corbett, T.K. Mal, Environ. Pollut. 120 (2002) 319-323.

[15] Uwah E.I., N.P Ndahi, V.O. Ogugbuaja, J. Appl. Sci. Environ. Sanit. 5 (2009) 83-90.

[16] Wang H.H., X.Q. Shan, B. Wen, G. Owensb, J. Fang, S.Z. Zhang, Environ. Exp. Bot. 61 (2007) 246-253.

[17] Wensheng S., L. Chonytu, Z. Zhiquan, Chinese J. Appl. Ecol. 8 (1997) 314-318.

[18] Yilmaz K., I.E. Akinci, S. Akinci, New Zealand J. Crop Hortic. Sci. 37 (2009) 189-199.

[19] Yoshida S., D. Fordo, J. Cock, K. Gomez (1972). Laboratory manual for physiological studies of rice $3^{\text {rd }} \mathrm{Ed}$.

[20] Williams and Twine (1960). In: Modern methods of plant analysis, pp. 3-5.

[21] T. Mahakavi, R. Bakiyaraj, L. Baskaran, Nusrat Rashid, K. Sankar Ganesh, International Letters of Natural Sciences 4 (2014) 58-65.

[22] B. Elayaraj, International Letters of Natural Sciences 12(1) (2014) 85-93. 